\title{
Les « émeutes de la faim »: une lecture (géo-politique) du changement (social)
}

\author{
Pierre Janin \\ Chargé de recherches IRD \\ UMR 201 «Développement et sociétés » \\ IEDES (Université de Paris I) - IRD \\ 45bis, , avenue de la belle Gabrielle \\ 94736 Nogent sur Marne cedex \\ Mél : pierre.janin@ird.fr
}

En 2008, la faim a fait un retour brutal dans les agendas politiques et médiatiques internationaux et nationaux. Doit-on cependant rappeler qu'elle touche, chaque année, plus de 850 millions de personnes dans le monde? Avec elle, ce sont les peurs ancestrales, jamais vraiment apaisées, des sociétés (comment se nourrir ?) et des gouvernants (comment les nourrir ?) qui se réveillent. De fait, la facture alimentaire a été difficile à honorer en 2008, pour les familles comme pour les gouvernants. Au fond, elle a servi de révélateur de la vulnérabilité de l'économie de marché et des compromis, nationaux comme internationaux, qui assuraient une certaine cohésion, à défaut de régulation véritable. Elle a montré aussi que les perdants (les pays pauvres à déficit vivrier, importateurs et dotés de peu de ressources) et les gagnants de la crise (pays agro-exportateurs soutenus par de vigoureuses politiques incitatives) ne pouvaient faire cavaliers seuls. Désormais, l'alimentation possède, pour ceux qui en doutaient encore, une indéniable dimension géo-politique.

Au-delà de leur dimension alimentaire (comment acheter ce qui est rare et cher lorsqu'on est pauvre ?), les manifestations sociales, parfois violentes, qui ont émaillé le printemps 2008 ont des racines plus profondes (Ouedraogo 2008) ${ }^{1}$.

Interpelés vivement par une frange active des populations urbaines paupérisées, les pouvoirs politiques nationaux ont, en effet, parfois été lents à réagir (Cameroun, Sénégal), pariant sur l'essoufflement du mouvement ; d'autres, au contraire, ont été prompts à saisir le désarroi ambiant réactivant certains ferments nationalistes. C'était sans compter l'effet de contagion et de relance médiatique généré par des médias, avides d'images et de formules chocs (Janin 2008a).

Quels enseignements tirer des événements sociaux dont la paternité a été attribuée, parfois de manière un peu hâtive, à ce que l'on a appelé " la crise alimentaire »? Quelles en sont les causes immédiates et plus lointaines? Quels en sont les auteurs? Ce texte n'entend pas faire un bilan détaillé de la crise mais plutôt fournir quelques clés de compréhension du changement qu'il dévoile et précipite.

\section{Une crise alimentaire à facettes}

L'année 2008 a été marquée par une très grande instabilité des marchés mondiaux. Les prix des matières premières agricoles, comme des dérivés énergétiques, ont d'abord atteint des sommets au printemps 2008 avant de décroître de manière rapide à l'automne. C'est cette tension mondiale de l'offre et de la demande - annoncée par certains (la FAO entre autres), considérée comme excessive par d'autres ${ }^{2}$, pour certaines denrées alimentaires de base, qui a constitué «la crise alimentaire » comme objet.

\footnotetext{
${ }^{1}$ Entre janvier 2007 et avril 2008, 37 pays ont été touchés, surtout en Afrique (Cameroun, Burkina, Maroc, Egypte, RCl, Mauritanie, Sénégal, Afrique du Sud) mais aussi en Bolivie, au Mexique, au Bangladesh, au Pakistan, au Yémen, en Ouzbékistan.

${ }^{2}$ La hausse tendancielle des prix agricoles mondiaux amorcée depuis le début de l'année 2007 (après 30 ans de bas prix, favorable aux consommateurs) la rendait prévisible même si en prix constants, ils étaient inférieurs à ceux de 1974-1975 et de la crise pétrolière.
} 
Les réactions anticipées à certains phénomènes conjoncturels (baisses locales des récoltes, diminution des stocks mondiaux) ont également démultiplié les effets (loi de King). En réalité, cette inflation n'a véritablement touché que la part réduite des céréales (riz, blé, maïs) faisant l'objet d'échanges internationaux ${ }^{3}$. Qui plus est, la transmission de cette hausse des prix aux marchés domestiques s'est effectuée de manière très inégale et décalée. En parallèle, les prix des céréales locales (mil, sorgho, maïs) - faisant l'objet d'une marchandisation importante et ancienne à destination des villes et des pays côtiers - ont eux-mêmes connu une hausse marquée, dans la plupart de capitales africaines alors même qu'aucune pénurie d'envergure n'était annoncée ni relevée. Puis, très vite, dès le mois de juillet 2008, l'indice des prix céréaliers mondiaux s'inversait : après avoir culminé à 281 en février 2008, il retombait autour de 175 en décembre (à un niveau assez proche de la fin de l'année précédente). Ce repli ne signifie pas pour autant une rémission rapide des difficultés alimentaires d'approvisionnement pour les pays (et les catégories) pauvres touchés. Qui plus est, si l'idée d'une régulation mondiale fait son chemin (des stocks? Un fonds?), elle n'obère pas le risque d'instabilité.

L'année 2008 s'est achevée avec une progression sensible de l'insécurité alimentaire (+ 44 millions de personnes pour culminer à environ 967 millions d'habitants contre 825 millions en 2004). Au demeurant, la FAO estimait, en avril 2008, que la facture alimentaire pour les pays pauvres à déficit vivrier avait augmenté de 58\% depuis le mois de janvier, après une hausse de 37\% en 2007.

Ces quelques remarques préliminaires tendent à prouver que la notion de «crise alimentaire » est ambiguë (Granier 2009). Ce phénomène mondialisé doit, en effet, être décomposé en autant de sousobjets que de schémas explicatifs.

Le premier définit la crise de 2008 comme une rupture de tendance sous l'effet d'un ou de plusieurs chocs, subits et exogènes. Dans cet ordre d'idée, elle serait donc un phénomène importé, dont la hausse des prix serait, à la fois, la cause et le résultat. Celle-ci aurait pour effet de favoriser chez les producteurs agricoles, les consommateurs ruraux et urbains et les décideurs politiques, les explications déterministes. Les responsables auraient le mérite d'être faciles à identifier : aléas climatiques (sécheresse et inondations selon les régions) d'une part, actions spéculatives des fonds d'actions sur les commodities. Autant de "boucs émissaires » commodes permettant de réduire la complexité de la crise et de mettre en œuvre certaines rhétoriques dénonciatrices (vis-à-vis des entreprises agro-alimentaires ou de la FAO par exemple) de nature, par exemple, à détourner l'attention des opinions publiques nationales quant à l'absence de politiques agricoles dignes de ce nom $^{4}$ depuis plusieurs décennies.

Le deuxième considère la crise alimentaire comme le résultat d'une rupture des grands équilibres, de l'offre et de la demande, mais pas seulement. Elle exprimerait plus fondamentalement une fragilisation de la résilience des systèmes productifs et marchands sur fonds d'explosion de la demande de consommation céréalière. Les premiers ne disposant plus de marges de manœuvres suffisantes en terme de productivité : le décalage entre potentialités agricoles et ressources extraites allant de manière croissante ; les seconds ayant délaissé la fonction essentielle de stockage - qui permettait traditionnellement d'amortir les effets des crises localisées dans le temps et dans l'espace - pour fonctionner à flux tendus ${ }^{5}$. Ceux qui soutiennent cette thèse possibiliste auraient donc une vision plus réactive et plus alarmiste. Dans cette mouvance, resurgit le vieux débat entre agricultures familiales vivrières et monocultures d'exportation, sous une forme actualisée : la production d'agrocarburants de première génération serait la principale cause de la hausse des prix alimentaires depuis $2002^{6}$.

Le troisième, enfin, envisage la crise alimentaire comme un système complexe, multi-échelles et multi-acteurs, au carrefour des champs sociaux, économiques et politiques, mettant à mal l'ensemble des scenarii établis. Cette conception a quelque fondement si l'on relève les difficultés éprouvées par les experts pour saisir toutes les ramifications de la crise alimentaire, aggravée par la crise

\footnotetext{
$312 \%$, soit environ 258 millions de tonnes sur 2.120 millions utilisées dans le monde en 2007-2008.

4 Entre 1985 et 2005, l'aide publique au développement agricole, destinée à l'Afrique subsaharienne, est passée d'environ 3,2 milliards de dollars à 1,1 milliard de dollars.

${ }^{5}$ En sept ans, les stocks de riz blanc ont été divisés par deux, passant de 147 millions de tonnes en 2000 à 71 millions en 2007. Hors riz, les stocks céréaliers ne dépassaient pas 230 millions de tonnes en 2008 contre 420 millions dix plus tôt alors que la consommation est passée, dans le même temps, de 1.450 millions à 1.700 millions de tonnes.

${ }^{6}$ Même si l'utilisation industrielle des céréales augmente, elle ne dépassait pas 100 millions de tonnes en 20072008 dont 95 millions de maïs).
} 
énergétique et financière. Qui peut, en effet, se targuer d'avoir prédit une si grande volatilité des prix alimentaires (à la hausse puis à la baisse) ? Comme si par une sorte d'ironie, le perfectionnement des modèles prédictifs du risque ${ }^{7}$ ne parvenait pas à lever le champ des incertitudes. Ainsi conçue, la crise alimentaire fonctionnerait comme un objet autonome, celle de l'accumulation de péjorations et de dysfonctionnements structurels dont on ne découvrirait que tardivement les interactions et les rétroactions négatives. En effet, que penser des replis protectionnistes que la crise aura favorisé : le marché mondial du blé s'est rétréci d'un tiers et la situation était encore pire pour le riz ${ }^{8}$ ? Parallèlement, d'autres ont semblé (re)découvrir les dérives de modèles agricoles basés la spécialisation culturale des aires géographiques, selon la théorie des avantages comparatifs, dont la mise sous tutelle de vastes emprises foncières (à l'instar des grandes plantations coloniales) ne serait qu'un des derniers avatars, au détriment d'une autosuffisance alimentaire minimale. Que penser également de la diffusion de modèles alimentaires, valorisant socialement la consommation de viande, fortement consommatrice des céréales?

\section{La rupture des capacités et des compromis nationaux}

Les «émeutes » de l'année 2008 ne renvoient pas à des situations de pénuries alimentaires aggravées, comme dans les zones de conflit ou lors des épisodes de grande sécheresse. Hormis certaines ruptures conjoncturelles d'approvisionnement ${ }^{9}$, les denrées de base n'ont, dans l'ensemble, pas fait défaut sur les marchés urbains.

Ce qui est en cause dans cette crise, c'est la capacité propre des ménages urbains à se nourrir, puisque tous les apports extérieurs (revenus de la migration, aide vivrière des ruraux) ont été revus à la baisse. Et surtout celle des classes moyennes, jusque-là épargnées par une insécurité alimentaire essentiellement considérée comme rurale. Ce sont eux qui ont été les plus vivement touchés. N'ayant habituellement pas de difficultés majeures pour s'alimenter, cela explique leur très fort sentiment de déclassement social et de désarroi. Tous les entretiens réalisés à Bamako et à Ouagadougou en font état. La réaction des émeutiers se fonde donc en partie sur la brutalité de la perte de pouvoir d'achat, même si le processus de paupérisation est bien antérieur.

On a acheté plus que jamais «au détail » (un kilo de riz, un petit tas de tomate, quelques morceaux de viande avec os) et non plus «en sac » pour stocker. Parce qu'on « n'a plus les moyens » certes, mais aussi parce que ceux qui le font sont souvent dans l'obligation d'aider les frères et sœurs « moins bien placés » et les « parents de village ».

Dans les faits, les diminutions ont surtout été sélectives et qualitatives. Elles ont porté sur la viande, le poisson, les légumes frais, les boissons et rarement sur les céréales de base (riz, maïs). On a beaucoup plus rarement réduit l'argent quotidiennement consacré à la « popote ». En revanche, on a parfois mise en réserve de la nourriture pour le lendemain. C'est à l'occasion des fêtes religieuses (Mouloud, Tabaski, Noël,...) et familiales que la perte de capacité a été la plus frappante. Et dans cette bataille alimentaire, chefs de ménage et épouses étaient bien seuls. Comme si le contrat social tacite qui les liait à l'Etat avait été rompu, après celui qui faisait de la famille un amortisseur social.

Depuis plusieurs décennies, la tendance générale, en effet, a été de favoriser l'approvisionnement alimentaire des citadins, via des politiques de subventions aux prix des produits importés et de prix peu rémunérateurs pour les productions agricoles locales. Le choix a parfois été fait de se fournir sur le marché mondial, là où se trouvaient les excédents les moins chers, quitte à fragiliser certaines filières nationales (riz, maïs). Cette situation reste cependant marginale et il convient de relever la capacité renouvelée des agricultures familiales à fournir les marchés, dans un contexte de croissance rapide de la demande. On a alors pendant longtemps parlé pudiquement, de «biais urbain » pour désigner ces compromis préférentiels. On peut aussi considérer qu'il s'agissait là d'une " cotisation politique » destinée à préserver les lieux centraux du pouvoir de toute contagion sociale d'envergure.

\footnotetext{
${ }^{7}$ Ces modèles sont essentiellement « positivistes », car basés sur les acquis de la science ; « quantitatifs » car adossés à des outils de mesures et de prévisions ; « réductionnistes » car se focalisant plutôt sur les aspects techniques en l'absence des actions humaines et négligeant les interactions et les champs de causalités; " technocratiques », car nourris par les dires d'experts et les règles de l'auto-évaluation.

${ }^{8}$ Chine, Indonésie, Vietnam, Égypte, Inde et Cambodge ont interdit ou strictement limité les exportations. Le Kazakhstan, la Russie, l'Ukraine et l'Argentine ont mis en place des interdictions ou des restrictions d'exportation sur le blé.

${ }^{9}$ En riz notamment, au Sénégal et au Mali, les grands importateurs ayant eu parfois du mal à boucler certains contrats, faute de pouvoir obtenir les lignes de crédit nécessaires.
} 
Mais, à partir des années 1990, les politiques de libéralisation sont progressivement saper idéologiquement et financièrement les instruments d'intervention (et de la régulation) étatique et faire voler en éclats ce contrat social.

Elles ont constitué, à bien regarder, une violence économique et sociale dont il est difficile de nier les effets déstabilisants à moyen terme. Les familles ont ainsi expérimenté des politiques marquées par des ajustements à la baisse des salaires, des licenciements. Les Etats, sommés de réduire leur train de vie et leurs dépenses, de se désendetter, ont peu à peu délaissé des pans entiers de l'économie et de la société (mesures de soutien alimentaires, appuis incitatifs agricoles, subventions aux prix,...). On a privatisé des sociétés agro-exportatrices, des entreprises para-publiques (pour l'eau et l'électricité par exemple), fait disparaître les Caisses de stabilisation (qui avaient certes échoué à soutenir les prix agricoles aux petits producteurs), refondu les missions des Offices Céréaliers...

La hausse très forte et rapide des prix des produits de première nécessité entre 2007 et 2008, semble avoir cristallisé frustration, colère et désarroi qui préexistaient. C'est la conjonction de plusieurs phénomènes (baisse du pouvoir d'achat, de la capacité d'entraide familiale et de la « débrouillardise ») qui est à l'origine de cette flambée de violence prévisible. C'est pourquoi, la baisse mesurée des prix de détail, depuis le mois de juillet 2008, n'a pas été en mesure de réduire l'insécurité alimentaire. De nouveaux épisodes revendicatifs ne sont donc pas à exclure...

\section{Les procédures de « mise sous tension »}

Les tensions sociales liées à des situations de pénurie alimentaire ne sont pas nouvelles dans les "pays pauvres à déficit vivrier » d'Afrique subsaharienne. Disettes, soudures alimentaires et crises localisées y présentent un caractère routinier, presque banalisé depuis les périodes précoloniales. L'histoire des Sahels est marquée par la succession d'excédents sporadiques et de pénuries fréquentes. Pour autant, elles ont rarement donné lieu à des manifestations violentes. Sans doute, parce qu'elles touchaient davantage des populations rurales, dispersées, éloignées des centres de décision et plus enclines à endurer le manque ${ }^{10}$. Ce qui est nouveau, ce sont les protestations urbaines qui se sont exprimées dans les pays dotés de meilleures potentialités agricoles. Quels ont donc été les catalyseurs de ce qui a été médiatisé sous le terme « d'émeutes urbaines de la faim »?

Rappelons tout d'abord quelques caractéristiques micro-sociologiques des grandes villes. Un afflux régulier et massif de ruraux les a fait croitre, de manière rapide, au-delà de leur capacité d'absorption : l'emploi comme le logement y sont rarement assurés dans les conditions souhaitées. Ces immigrants sont généralement jeunes, peu diplômés, sans capital (financier ou social) pour acquérir une autonomie véritable à court terme. Quant aux «migrants de retour", partis "se chercher » dans d'autres territoires, ils sont souvent en errance dans leur propre pays. Beaucoup d'entre eux viennent s'installer dans des zones d'habitat non loti, sans eau, ni électricité, souvent dénués de services publics de proximité, en deuxième ceinture des villes. A l'étalement urbanistique répond aussi la fragmentation sociale de ces unités de logements qui constitue un important facteur de vulnérabilité. $Y$ résider impose à leurs occupants des déplacements journaliers, longs, aléatoires et éprouvants.

Nombre d'individus luttent, chaque jour, pour gagner l'argent indispensable à leur survie, sans savoir s'ils y parviendront, ni a fortiori quel en sera le montant. A tel point que, même avec une rémunération modeste ${ }^{11}$, un salarié pourra faire figure de privilégié. Pour beaucoup, la quête de sécurité l'emporte sur le niveau du gain monétaire parce qu'il permet d'élargir l'horizon.

Par ailleurs, ne pas être en mesure de s'approvisionner pour se nourrir n'est pas un phénomène nouveau dans les villes africaines. Avec l'équivalent d'1 ou 2 euros par jour, de très nombreux ménages doivent à la fois se nourrir, se déplacer, se soigner et éduquer leurs enfants. Ce qui implique des choix drastiques, et parfois des renoncements, en termes de priorités essentielles.

Dans un tel halo d'incertitudes et de précarités, il paraît difficile aussi de « se construire » comme acteur de sa propre vie. Chacun peut être alors tenté de se débrouiller seul, affaiblissant un peu plus la cohésion, la confiance (Akindès 2007) et la capacité de gestion du ménage. En ville, on ne réussit

\footnotetext{
${ }^{10} \mathrm{~L}$ 'éthos de la frugalité comme l'abnégation fataliste (mise en avant comme une vertu religieuse) des sociétés sahéliennes participent ainsi à cette construction sociale.

${ }^{11}$ Le salaire minimum des conventions collectives ne dépasse actuellement pas 40.000 francs CFA (soit environ 60 euros) en Afrique de l'Ouest.
} 
plus « pour » et «avec » sa famille comme auparavant et le sentiment de réussite ou d'échec est de plus en plus individuel. Cette évolution est le résultat du «travail » qui s'accomplit dans des sociétés, de plus en plus ouvertes, confrontées à la globalisation des espérances, des revendications et des comportements.

A cet égard, les effets d'annonce, les promesses non tenues, l'enrichissement rapide et illicite, le régime d'impunité qui règne souvent, pèsent aussi lourd que le prix du riz et le manque d'argent ! Elles contribuent à nourrir le désenchantement des jeunes et expliquent leur errance géographique et psychologique.

Cette réalité urbaine constitue en quelque sorte ce que l'on pourrait appeler "la procédure de mise sous tension des individus ". Comme le déclarait un infirmier burkinabè, père de famille, interrogé en mars 2009 à Ouagadougou : "dès le 5 du mois, je suis un ras le bol ${ }^{12}$. Elle conduit chacun à un incessant va-et-vient réflexif entre espoirs déçus et précarités vécues. Tandis que la juxtaposition spatiale et la confrontation visuelle entre des enclaves résidentielles de standing et des ilots d'habitat précaire et dégradé constituent un indéniable ferment de contestation. Ainsi, la ville est peu à peu devenue le territoire vécu des inégalités et de l'indifférence (Janin 2001), à la différence du « village » où l'on est rarement seul et exclu.

Par ailleurs il convient aussi de ne pas sous-estimer l'effet de contagion de ceux, qui «partis en migration » au Nord, reviennent régulièrement en visite et participent à la construction d'imaginaires biaisés de leur propre richesse. Dans le même ordre d'idée, le sentiment de pauvreté et de vulnérabilité est également renforcé, par les médias qui mettent en exergue les exemples d'accumulation rapide, parfois à bon compte, de quelques individus (Banegas et Warnier 2001). C'est pourquoi, le sentiment de pauvreté pèse d'un plus grand poids moral et politique en ville qu'en milieu rural.

Autant de comparaisons qui favorisent l'apparition de frustrations, de ressentiments qui ne font pas véritablement l'objet de régulation comme ils pouvaient l'être communément au village. Dans ces villes africaines, chacun est souvent désormais confronté à lui-même et peine à pouvoir mobiliser des appuis et soutiens en cas de difficulté supplémentaire. "Prie Dieu pour ne pas être riche parmi les pauvres " s'intitule, fort à propos, un documentaire récent sur la place et le rôle des grands commerçants tchadiens. A tel point que l'on préfère parfois déménager pour mettre une certaine distance avec la famille ou que l'on ignore (parfois sciemment) jusqu'au nom de ses voisins en difficulté, dans la cour d'à-côté.

\section{La politisation de la faim et ses mises en scène}

Avec cette crise, l'alimentation a véritablement émergé comme enjeu global et planétaire. A chaque échelle géographique, se sont révélées, de manière criante, les inégalités de développement, la violence des rapports de force, l'inefficacité des transferts, la vulnérabilité des sociétés et des Etats. Indirectement, cette crise a aussi montré comment les politiques de développement participaient à la construction de ce risque. A mesure que s'accroissent les phénomènes d'interdépendance, la sécurité alimentaire apparaît de plus en plus comme incertaine et fuyante.

Certes, la gestion de la faim a toujours été un enjeu sociétal et politique majeur. Mais, il semble bien que l'on assiste, depuis une décennie, à une (re-)politisation de la question alimentaire (Janin et Suremain 2005), alors même que la lutte contre la faim se professionnalisait et se technicisait (dans ses outils). Dans cette évolution, l'émergence et la réappropriation du concept de souveraineté alimentaire par les «acteurs sociaux émergents " (mouvements altermondialistes, association de défense des consommateurs, coalitions contre la vie chère, fédérations paysannes, syndicats, ONG locales,...) compte pour beaucoup.

En réaffirmant que "la faim est l'une des pires violations de la dignité humaine » (K. Annan 2002) et que l'alimentation est un droit inaliénable (passage quelque peu oublié de la Déclaration des droits de l'homme de 1948), on participe indéniablement à la construction des représentations dichotomiques et normatives des crises alimentaires. Celles-ci sont de nature à favoriser les postures de victimisation (nous, les pauvres, les pays du Sud) comme de dénonciation, voire de diabolisation (eux, les riches, les nantis, les multinationales, les commerçants importateurs, les pays du Nord, les institutions internationales). On fournit, dès lors, un argumentaire susceptible de nourrir d'autres

12 Où comment des individus sont poussés à bout, en raison de leur incapacité à faire face aux besoins élémentaires. 
formes de ressentiment et d'expression populaires violentes. " Débrouiller n'est pas voler » disent les jeunes africains. De là, à penser que le pillage est une compensation, il n'y a qu'une nuance contextuelle.

Par ailleurs, la crise alimentaire a également fourni, à certains gouvernants, une scène politique (Janin 2008b) : promettant aides et secours à « leurs populations ", vantant les mérites de leur "diplomatie alimentaire", incitant à «produire plus et manger local», dénonçant les « commerçants spéculateurs ». Certains en ont retiré des gains de popularité qui dépassent sans aucun doute l'efficacité réelle des quelques mesures prises (suspension de droits de douane à l'importation, gel des prix de détail, aide alimentaire,...).

Une autre explication des émeutes pourra être recherchée du côté des « effets d'aubaine sociale et politique » procurés par la diffusion des images de la faim et des revendications. L'insistance médiatique à montrer les multiples effets de la crise alimentaire ont sans doute pesé, accentuant le sentiment d'insécurité et d'impéritie des Etats et des institutions auprès des populations. Elle a, de manière assez délétère, créé un effet de contagion à l'intérieur des Etats et entre Etats, permettant l'agglutination des mobilisations citoyennes.

Il y a donc eu un effet de cristallisation du ressentiment social, accumulé depuis de longues années, et qui travaillait les sociétés urbaines en arrière plan. La crise alimentaire a donc servi de « défouloir » aux formations sociales émergentes (De Boeck et Honwana 2000), d'autant plus que le déficit démocratique de certains régimes devenait pesant (Delcourt 2009). Les «jeunes", catégorisation bien incertaine en Afrique, qui ont eu le sentiment d'être des générations sacrifiées et d'être mal représentées politiquement, se sont fortement mobilisés. Ce qui n'a rien d'étonnant si l'on tient compte aussi de l'accroissement des inégalités, allant parfois jusqu'à l'ostentation. La foi religieuse qui permettait de sublimer les situations de pauvreté, de précarité ou de faim, semble ellemême avoir de la peine à opérer. Enfin, sur fonds de mobilisation citoyenne (ouverture politique), le sentiment d'injustice sociale, l'idée d'autisme politique et d'inamovibilité excessive des dirigeants et des élites enflent (Kenya et Cameroun).

Il faut souligner le fait que certaines catégories de citadins (les ménagères), qui ne manifestent généralement pas ont, cette fois, participé à ces émeutes. C'est un élément nouveau à prendre en compte. En revanche, les plus pauvres sont restés hors-jeu : la «vie chère » n'a pas véritablement été ressentie faute de pouvoir acheter des denrées au marché et « préparer » (cuisiner un plat)!

Ces épisodes de crise jouent donc un rôle cathartique et politique évident : c'est autant le sentiment de soi qui s'éprouve que la découverte d'une manière d'être ensemble. A cet égard, comme dans les conflits civils, la crise alimentaire a servi de galop d'essai aux plus jeunes générations pour se faire connaître et entendre. Elle implique donc que les gouvernants s'intéressent de plus près à la manière dont on survit en ville afin de tenter de mieux anticiper les futures crises.

\section{Conclusion}

Depuis plus d'une année, experts et chercheurs n'en finissent pas de débattre sur les origines et les effets de la crise alimentaire (Dufumier et Hugon 2008). Et les enjeux ne sont pas des moindres puisqu'il s'agit rien moins de que (re)penser le développement et les politiques qui vont avec.

Si la crise alimentaire est sortie de l'agenda médiatique et politique, laissant la place à ses corollaires financiers, monétaires et bancaires, elle reste plus que jamais d'actualité pour la moitié des ménages africains qui vivent avec moins d'un euro par jour.

Tout bien considéré, l'exemplarité de cette crise réside dans la conjonction de trois formes d'insécurité alimentaire : un décalage entre besoins et ressources (manque de disponibilités), un décalage entre besoins et capacités (difficultés d'accès monétaire) et un décalage entre besoins et droits (affaiblissement des filets de sécurité). C'est donc en quelque sorte une crise réenchassée dans des situations à risque plus complexes (Delcourt 2008).

Les « émeutes de la faim » montrent la défiance croissante des populations appauvries vis-à-vis de l'ensemble des institutions étatiques et internationales pourtant appelées à la rescousse. Elles illustrent aussi la capacité de mobilisation populaire, parfois spontanées (les collectifs « contre la vie chère », les « défilés de ménagères »), parfois relayées (par les mouvances syndicales et politiques 
d'opposition). A cet égard, elles inaugurent bien une crise de gouvernementalité et de légitimité dont il faudra, cahin-caha aussi, tirer les leçons.

Au-delà de la bataille sur les modèles explicatifs de la crise - qui a opposé les experts et passionné les opinions publiques -, les « événements » peuvent aussi être interprétés comme le signe d'un désarroi sociétal, mélange détonnant de lassitude et de perte de repères, face à un environnement instable et complexe, dont il faudra tenir compte.

\section{Bibliographie}

Francis Akindès, 2007, "Pauvreté et construction des droits en Afrique "? West African Review, $n^{\circ} 10$ (http://www.westafricareview.com).

Richard Banégas et Jean-Pierre Warnier, 2001, « Nouvelles figures de la réussite et du pouvoir », Politique africaine, $\mathrm{n}^{\circ} 82$, pp. 5-21.

Filip De Boeck et Alcinda Honwana, 2000, Enfants, jeunes et politique, Politique africaine, $\mathrm{n}^{\circ}$ 80, pp. 5-110.

Laurent Delcourt, 2008, Mobilisations dans le Sud face à la crise alimentaire, in : Etat des résistances dans le Sud - 2009. Face à la crise alimentaire, 228 p. (http://www.cetri.be/spip.php?article970).

Issa Serge Coelo et Claude Arditi, 2006, «Prie Dieu pour ne pas être riche parmi les pauvres », $58 \mathrm{mn}$. Prod. Parenthèses Film, Paris, .

Marc Dufumier et Philippe Hugon, 2008, Les « émeutes de la faim » : du sous-investissement agricole à la crise sociopolitique », Revue Tiers-Monde, n`196, pp. 927-934.

Jean-Maxence Granier, "Sémiotique de la crise", Think-Out, 18 février 2009 (http://www.thinkout.fr/IMG/pdf_Semiotique-de-la-crise-JMG-2009-2.pdf).

Pierre Janin, 2001, « Une géographie sociale de la rue africaine », Politique africaine, n 82, pp. 177-189.

Pierre Janin, 2008a, «Crise alimentaire mondiale. Désordres et débats », pp. 9-13, in : Janin P. et Giblin B., 2008. (éds.), Les enjeux de la crise alimentaire mondiale, Paris, La Découverte, Hérodote, $\mathrm{n}^{\circ}$ 131, $206 \mathrm{p}$.

Pierre Janin, 2008b, «La mise en scène de la lutte contre l'insécurité alimentaire au Mali : discours, postures et jeux d'acteurs ", IRD, Dakar, 26 p. (http://hal.ird.fr/ird-00332050/fr/).

Pierre Janin et Charles-Edouard de Suremain, 2005, La question alimentaire en Afrique : risque et politisation, Paris, Puf, Revue Tiers-Monde, $\mathrm{n}^{\circ}$ 184, pp. 727-859.

François de Charles Ouedraogo, 2008, "Vie chère : les causes apparentes et cachées », Le Faso.net, 29 avril 2008 (http:/ /www.lefaso.net/spip.php?article26606\&rubrique3) 